\title{
Oxygen Diffusion in Glassy Poly(ethyl methacrylate): Spatial Correlation of Jump Rates
}

\author{
Vladimir M. Syutkin*, Vladimir L. Vyazovkin, Sergey Grebenkin \\ Voevodsky Institute of Chemical Kinetics and Combustion, \\ Siberian Branch of the Russian Academy of Sciences, \\ Novosibirsk, 630090, Russian Federation
}

\section{Distribution Function of $Y_{n m}$}

Figure S1 demonstrates the distribution function of the random numbers $Y_{n m}$. The procedure of the generation of spatially correlated values $Y_{n m}$ is described in the article (Appendix A). The numbers $Y_{n m}$ have been generated using a simple cubic lattice with a size of $20 \times 20 \times 20$ sites. The total number of the $Y_{n m}$ used to build the distribution is equal to 24000. The distribution function is well described by the Gaussian function with zero mean and a variance which depends on $r_{c}$.

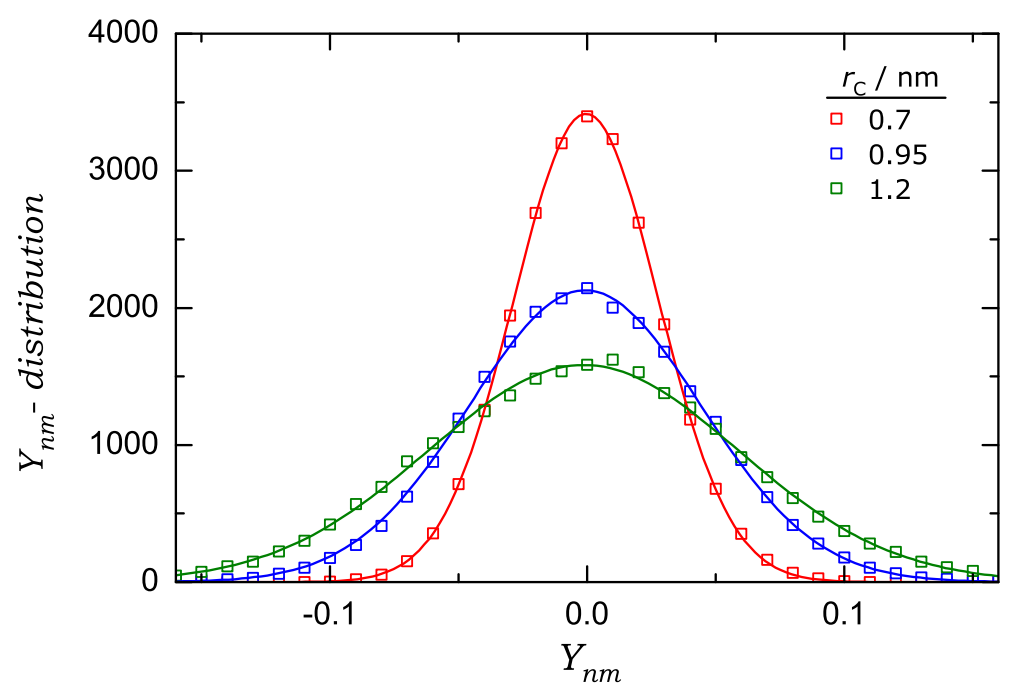

Figure S1: Symbols: number of numbers $Y_{n m}$ such that $0.01 \times I-0.005 \leq Y_{n m}<$ $0.01 \times I+0.005$, where $I=-16, \cdots, 16$. Lines are fits of $Y_{n m}$-distribution with the Gaussian function. The values of fitting parameter $r_{c}$ are given in the figure.

\footnotetext{
*syutkin@kinetics.nsc.ru
} 
Figure S2 demonstrates the spatial correlation function, $C F\left(r_{n m, p q}\right)$, for $Y_{n m}$. We found that the spatial correlation function can be well described by the equation

$$
C F\left(r_{n m, p q}\right) \equiv\left\langle Y_{n m} Y_{p q}\right\rangle=\left\langle Y_{n m}^{2}\right\rangle \exp \left(-r_{n m, p q}^{2} / 2 r_{c}^{2}\right)
$$

where the brackets $\langle\cdots\rangle$ denote averaging over all the pairs of lattice bonds separated by a distance $r=r_{n m, p q}$.

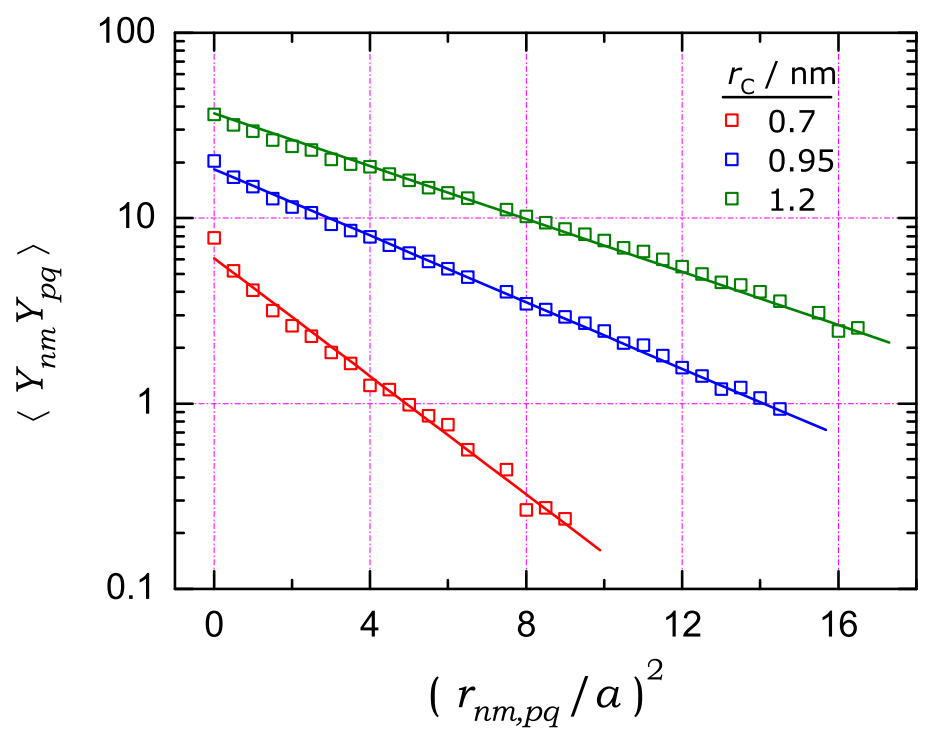

Figure S2: Symbols: the spatial correlation function, $C F\left(r_{n m, p q}\right)$, for $Y_{n m}$ which have been generated using a simple cubic lattice with a size of $20 \times 20 \times 20$ sites. The lines are fits to the equation $C F\left(r_{n m, p q}\right)=\left\langle Y_{n m}^{2}\right\rangle \exp \left(-r_{n m, p q}^{2} / 2 r_{c}^{2}\right)$. The values of fitting parameter $r_{c}$ are given in the figure. $a$ is the lattice constant.

\section{Dependence of $\sigma^{\ddagger}$ on temperature}

Figure S3 demonstrates the kinetic curves of nitrene oxidation in the temperature range from 90 to $118.5 \mathrm{~K}$ at oxygen concentration in polymer of $6 \times 10^{19} \mathrm{~cm}^{-3}$. The solid lines in the figure represents the kinetic curves simulated with the parameters $\left\langle F^{\ddagger}\right\rangle=15.2 \mathrm{~kJ} / \mathrm{mol}, \sigma^{\ddagger}=31.5 \times T \mathrm{~J} / \mathrm{mol}$ and $r_{c}=0.95 \mathrm{~nm}$. 


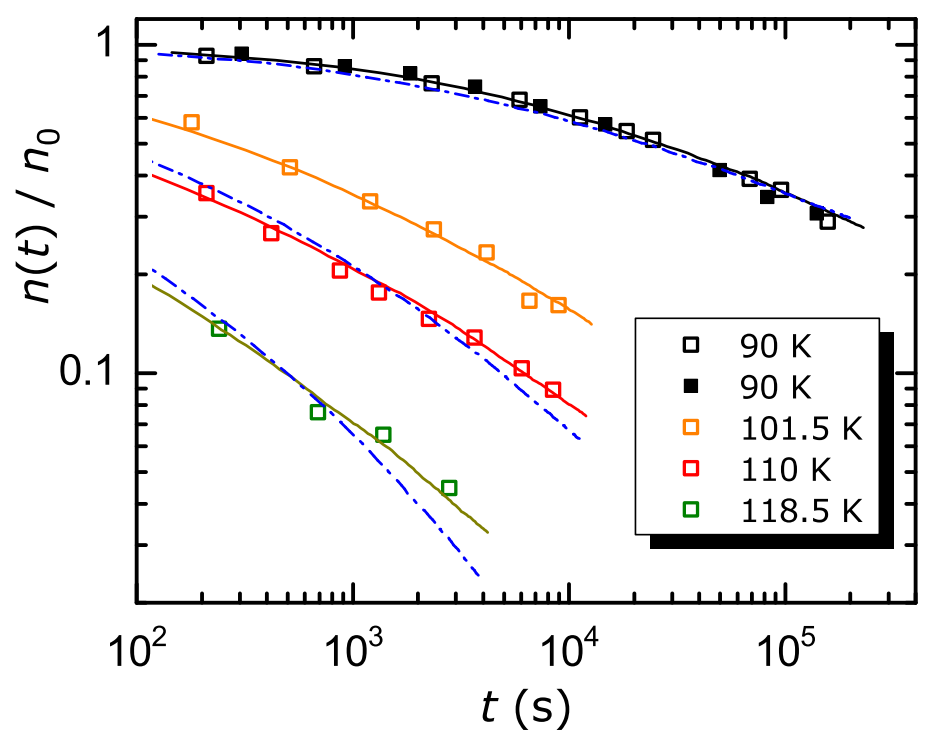

Figure S3: Symbols represent the oxidation curves of $p$-phenylazophenyl nitrenes in PEMA at oxygen concentration of $6 \times 10^{19} \mathrm{~cm}^{-3}$ at different temperatures; the data are taken from Syutkin et al. [J. Polym. Sci. Part B: Polym. Phys. 57, 1097 (2019)]. To demonstrate reproducibility, two kinetic curves measured at $90 \mathrm{~K}$ are shown. Solid lines were simulated using the following parameters of the presented model: $\left\langle F^{\ddagger}\right\rangle=15.2 \mathrm{~kJ} / \mathrm{mol}, \sigma^{\ddagger}=31.5 \times T \mathrm{~J} / \mathrm{mol}$ and $r_{c}=0.95 \mathrm{~nm}$. Dashed lines were simulated with the parameters $\sigma^{\ddagger}=3.2 \mathrm{~kJ} / \mathrm{mol}$ and $r_{c}=0.95 \mathrm{~nm}$ at all temperatures, $\left\langle F^{\ddagger}\right\rangle=15.2 \mathrm{~kJ} / \mathrm{mol}$ at 90 and $101.5 \mathrm{~K}$, and $\left\langle F^{\ddagger}\right\rangle=15.4 \mathrm{~kJ} / \mathrm{mol}$ at 110 and $118.5 \mathrm{~K}$. 